\title{
Parallel computing and the generation of basic plasma data
}

\author{
Vincent McKoy, ${ }^{\text {a) }}$ Carl Winstead, and Chuo-Han Lee \\ Noyes Laboratory of Chemical Physics, California Institute of Technology, Pasadena, California 91125
}

(Received 11 April 1997; accepted 24 August 1997)

\begin{abstract}
Comprehensive simulations of the processing plasmas used in semiconductor fabrication will depend on the availability of basic data for many microscopic processes that occur in the plasma and at the surface. Cross sections for electron collisions, a principal mechanism for producing reactive species in these plasmas, are among the most important such data; however, electron-collision cross sections are difficult to measure, and the available data are, at best, sketchy for the polyatomic feed gases of interest. While computational approaches to obtaining such data are thus potentially of significant value, studies of electron collisions with polyatomic gases at relevant energies are numerically intensive. In this article, we report on the progress we have made in exploiting large-scale distributed-memory parallel computers, consisting of hundreds of interconnected microprocessors, to generate electron-collision cross sections for gases of interest in plasma simulations. (C) 1998 American Vacuum Society. [S0734-2101(98)01001-X]
\end{abstract}

\section{INTRODUCTION}

Plasma reactors are used in $30 \%-40 \%$ of the processing steps in semiconductor fabrication. As the cost of semiconductor fabrication facilities escalates, so too does the research and development cost of introducing each new generation of plasma processing technology. It is now recognized that comprehensive three-dimensional plasma simulations are essential to reducing the need for the costly and time-consuming experimental characterization currently required to develop a new generation of processing equipment. ${ }^{1}$ While such simulations have generally been beyond the reach of conventional supercomputers, large-scale parallel computers now seem to have the potential to make them possible with industrially viable turnaround times. ${ }^{2}$

Improved simulations of plasma reactors will depend on the availability of basic data for many microscopic processes that occur in the plasma and at the surface. ${ }^{1}$ Cross sections for electron collisions, a principal mechanism for producing reactive species in the plasma, are among the most important data required, yet electron-collision data are at best sketchy, and often absent, for the polyatomic feed gases used in the plasmas of interest in microelectronics fabrication. Though among the most important, cross sections for electron-impact dissociation of molecules into neutral fragments are also among the most difficult to measure, while cross sections of any kind for transient species produced in situ may be experimentally inaccessible.

Computational approaches to obtaining such data are thus potentially of significant value. The collision of low-energy electrons with molecules is, however, a complex quantum mechanical problem. ${ }^{3}$ Calculating the probabilities of various possible outcomes of such a collision-that is, the scattering cross sections-is a correspondingly computationally intensive challenge. Hence, while several methods have been developed for numerical studies of such collisions, ${ }^{3-6}$ progress in their application to polyatomic gases used in the plasmas

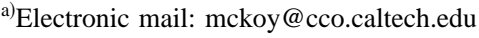

employed in semiconductor fabrication, e.g., species containing several carbon and halogen atoms, has been quite limited. Meeting the demand in simulations of these plasmas for electron-collision cross section sets for multiple species, e.g., feed gases, their dissociation fragments, and products of the etch process, requires not only computational methods of sufficient reliability, but implementations of those methods that can produce timely and cost-effective results. Such implementations, we believe, will rely on massively parallel computers, which can provide the absolute performance required by the scale of $a b$ initio calculations on polyatomics at a price that is not prohibitive.

In this article, we report on the progress we have made in exploiting large-scale distributed-memory parallel computers, consisting of hundreds of interconnected microprocessors, to generate electron-collision cross section data for gases of interest in plasma simulations. We begin with a brief overview of the variational principle employed in these calculations and a discussion of the significant computational demands of its application to polyatomic gases. We then outline the computational methodology that we have developed to exploit massively parallel computers to carry out the compute-intensive tasks in these calculations, and we briefly discuss the overall performance levels achieved in typical runs on the Intel Paragon (512 processors) and CRAY T3D (256 processors) parallel supercomputers. ${ }^{6-8}$ We then discuss results of applications of this procedure to several polyatomic species. We conclude with a few remarks on the impact that future generations of parallel supercomputers can have not only on our ability to obtain data for electron collisions and other microscopic processes needed in plasma simulations, but also on our ability to carry out comprehensive three-dimensional simulations themselves.

\section{FORMULATION AND IMPLEMENTATION}

At the low energies of interest in materials-processing plasmas, an accurate quantum-mechanical treatment of the full 3(N+1)-dimensional space of the Schrödinger equation 
that governs the collision of an electron with a molecule with $N$ electrons is necessary; low-order approximations, such as the Born approximation, are not applicable. Because direct integration of Schrödinger's equation in this $3(N+1)$-dimensional space is not practical, many recent theoretical studies have relied on the use of variational approximations to obtain the scattering amplitudes for these collisions.

In our studies, we use a multichannel extension of the variational principle for the scattering amplitude originally introduced by Schwinger. ${ }^{9}$ This Schwinger multichannel (SMC) method $^{10}$ is capable of addressing molecular targets of arbitrary symmetry, exchange interactions (indistinguishable particles), target polarization (distortion of molecular electron density by incident electron), and electronic excitation (multichannel problem). A key feature of the SMC method is that, as in Schwinger's original variational principle, the trial wave function need not satisfy scattering boundary conditions; in particular, square-integrable functions, such as the Cartesian Gaussians commonly used in molecular electronic structure calculations, may be employed.

Use of the SMC variational principle leads ${ }^{6,7}$ to a set of linear equations

$\mathbf{A x}=\mathbf{b}$,

where the matrices $\mathbf{A}$ and $\mathbf{b}$ incorporate all of the physical information about the molecule and the collision process. From the unknown matrix $\mathbf{x}$ we can determine a set of scattering amplitudes, complex numbers whose square moduli yield a set of cross sections. The elements of the complex symmetric matrix $\mathbf{A}$ have the form

$$
\mathbf{A}_{i j}=\left\langle\chi_{i}\left|\left(\frac{1}{N+1}-P\right) \hat{H}+V P-V G_{P}^{(+)} V\right| \chi_{j}\right\rangle,
$$

while those of the rectangular complex matrix $\mathbf{b}$ are given by

$$
\mathbf{b}_{i n}=\left\langle\chi_{i}|V| \Phi_{n} \exp \left(i \mathbf{k}_{n} \cdot \mathbf{r}_{N+1}\right)\right\rangle .
$$

In Eq. (2), $\chi_{i}$ and $\chi_{j}$ are Slater determinants of $(N+1)$ oneelectron orbitals in which the total scattering wave function is expanded, $P$ is a projection operator which projects the interaction-free Green's function, $G_{0}^{(+)}$, onto a finite number of energetically accessible channels, $G_{P}^{(+)}$is the projected version of $G_{0}^{(+)}, V$ is the interaction potential between the incident electron and the molecule, and $\hat{H}=E-H$ is the energy minus the total Hamiltonian operator. In Eq. (3), $\Phi_{n}$ is an electronic eigenstate of the molecule with energy $E_{n}$, and the plane wave $\exp \left(i \mathbf{k}_{n} \cdot \mathbf{r}_{N+1}\right)$ represents a free electron. The angle brackets in Eqs. (2) and (3) indicate integration over all $3(N+1)$ electron coordinates.

Construction of the matrices $\mathbf{A}$ and $\mathbf{b}$ requires the evaluation of several different types of integrals. If we expand the molecular orbitals in the Slater determinants $\chi_{i}, \chi_{j}$, and $\Phi_{m}$ in Eqs. (2) and (3) in Cartesian Gaussian functions

$$
\begin{aligned}
\zeta(\mathbf{r} ; \mathbf{R}, l, m, n)= & N_{\alpha}(x-X)^{l}(y-Y)^{m}(z-Z)^{n} \\
& \times \exp \left(-\alpha|\mathbf{r}-\mathbf{R}|^{2}\right),
\end{aligned}
$$

where $\mathbf{r}=(x, y, z)$ and $\mathbf{R}=(X, Y, Z)$, all integrals arising in the matrix elements of $\mathbf{A}$ and $\mathbf{b}$ can be evaluated analytically, except for those of $V G_{P}^{(+)} V$. Matrix elements of $V G_{P}^{(+)} V$ have no known analytical form and must be evaluated by numerical quadrature. Our quadrature scheme ${ }^{11}$ makes use of the spectral representation of the one-electron Green's function to write these $V G_{P}^{(+)} V$ terms in the form

$$
\left\langle\chi_{i}\left|V G_{P}^{(+)} V\right| \chi_{j}\right\rangle=\frac{1}{2 \pi} \lim _{\epsilon \rightarrow 0^{+}} \sum_{n \in \text { open }} \int d^{3} k^{\prime} \frac{\left\langle\chi_{i}|V| \Phi_{n} \exp \left(i \mathbf{k}^{\prime} \cdot \mathbf{r}_{N+1}\right)\right\rangle\left\langle\Phi_{n} \exp \left(i \mathbf{k}^{\prime} \cdot \mathbf{r}_{N+1}\right)|V| \chi_{j}\right\rangle}{E-E_{n}-\frac{1}{2} k^{\prime 2}+i \epsilon} .
$$

The matrix elements in the numerator of Eq. (5) give rise to one- and two-electron integrals involving Cartesian Gaussian functions and a plane wave, i.e.,

$$
\langle m \mid k\rangle=\sum_{\mu} \int d^{3} r \zeta_{m}(\mathbf{r}) \frac{Z_{\mu}}{\left|\mathbf{r}-\mathbf{R}_{\mu}\right|} \exp (i \mathbf{k} \cdot \mathbf{r})
$$

and

$$
\begin{aligned}
\langle m n \mid p \mathbf{k}\rangle= & \int d^{3} r_{1} \int d^{3} r_{2} \zeta_{m}\left(\mathbf{r}_{1}\right) \\
& \times \zeta_{n}\left(\mathbf{r}_{1}\right) \frac{1}{\left|\mathbf{r}_{1}-\mathbf{r}_{2}\right|} \zeta_{p}\left(\mathbf{r}_{2}\right) \exp \left(i \mathbf{k} \cdot \mathbf{r}_{2}\right),
\end{aligned}
$$

which can be evaluated analytically. ${ }^{12}$ Since we know how to evaluate these integrals, the $V G_{P}^{(+)} V$ term can now be constructed by doing the integrals over $\mathbf{k}^{\prime}$ by quadrature. Because this quadrature must be carried out over an infinite three-dimensional domain, tens of thousands of quadrature points may be required. At each quadrature point, all possible one- and two-electron integrals arising from a chosen set of Cartesian Gaussians must be evaluated. A very large number of integrals, particularly of the more numerous twoelectron class, may hence be required. In typical calculations with some hundreds of Cartesian Gaussians and of the order of $10^{5}$ quadrature points in $\mathbf{k}^{\prime}, 10^{10}-10^{13}$ integrals must be evaluated. Since of the order of $10^{2}$ floating point operations are required per integral, a very large computation is implied. Because studies of this kind are very demanding of computational resources, our progress has depended on exploiting the prodigious advances in computational power that have resulted from the development of massively parallel processors (MPPs).

The particular class of parallel computers we have used, which is also the class that has come to dominate the high- 
performance sector of the MPP field, is the distributedmemory, multiple-instruction, multiple-data design. Such a computer consists of a collection of computational nodes connected by a high-speed communications network to one another and to peripheral devices such as disk storage and ports for external connections. A node contains its own memory and one or more processing units (CPUs). Each CPU or microprocessor at a node is more or less the equivalent in computational power of a high-end workstation. The aggregate computational power and memory of several hundred to a thousand or more such nodes assembled into large MPPs are thus quite considerable.

The evaluation of a large number of the two-electron integrals over three Gaussians and a plane wave and the subsequent transformation to build the $\left\langle\chi_{i}|V| \Phi_{n} \exp \right.$ $\left.\left(i \mathbf{k}^{\prime} \cdot \mathbf{r}_{N+1}\right)\right\rangle$ matrix elements needed to obtain the $V G_{P}^{(+)} V$ term in $\mathbf{A}$ are the principal computational challenges in the SMC procedure. The labor involved in each of these steps grows rapidly with the size of the molecule: the integral evaluation scales as $N_{g}^{3} N_{k}$, where $N_{g}$ is the number of Gaussians and $N_{k}$ the number of $\mathbf{k}^{\prime}$ points in the quadrature in Eq. (5), while the transformation step is proportional to $N_{g}^{4} N_{k}$.

In fact, parallelizing the integral evaluation is straightforward: we simply assign each processor a different subset of integrals to evaluate. ${ }^{6}$ We have great flexibility in choosing this division of labor, as long as we obtain good load balance, i.e., as long as the work is shared equally. This step is perfectly parallel (involving no communication overhead). Combining the integrals is more challenging, since the rules for building the elements of $\mathbf{A}$ and $\mathbf{b}$ from these integrals are fairly complicated, and the CPUs must communicate with one another in this phase (because each CPU has in its own memory only a fraction of the batch of integrals in the MPP at any time). An efficient way of carrying out this step on a MPP is to fuse the rules for transforming the integrals over Gaussians into integrals over molecular orbitals with the rules for constructing the many-electron matrix elements in Eq. (5) from integrals over molecular orbitals, to build a single transformation matrix that, when multiplied with the current batch of integrals (arranged as a matrix), gives the necessary combinations as the product matrix. ${ }^{6,8,13}$ Each processor can build a block of this transformation matrix independently, so that the only communication among processors occurs during the matrix multiplication. In short, we have formulated both the evaluation and transformation of the vast array of integrals in terms of procedures that are either perfectly parallel or highly efficient and relatively easy to program (multiplication of large, dense, distributed matrices). The remaining step, solving $\mathbf{A x}=\mathbf{b}$ for $\mathbf{x}$, is straightforward.

The overall performance of our program on the Intel Paragon (512 processors) or the CRAY T3D (256 processors) cannot be characterized by a single number. Although evaluating and subsequently combining the integrals are by far the dominant steps in the calculation, the relative importance of these two steps is strongly dependent on factors such as the size of the molecule and the number of possible outcomes (channels) being considered in a collision. Depending on the

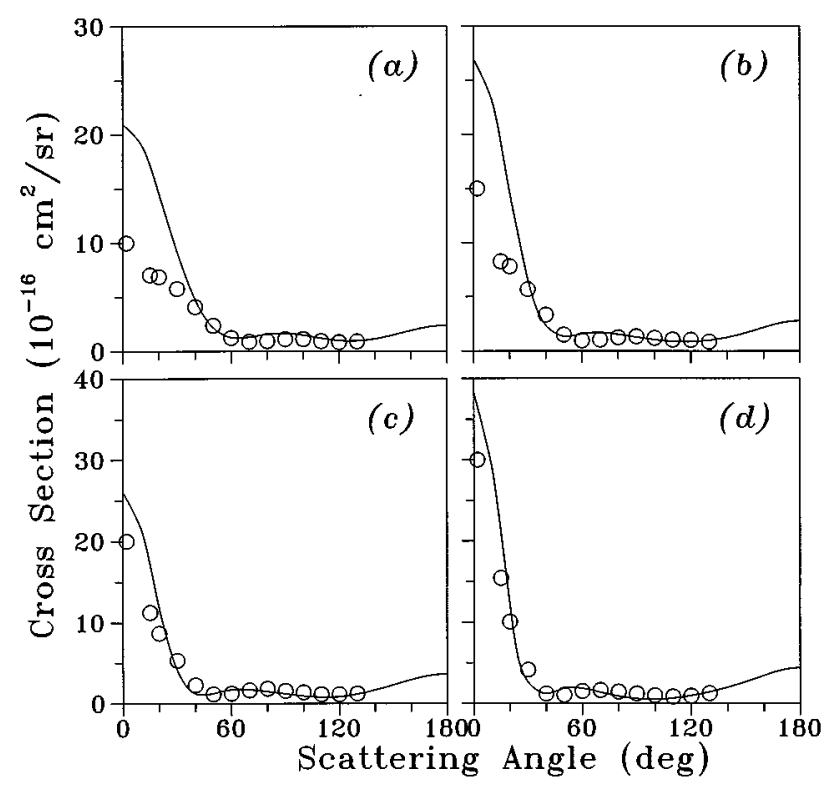

FIG. 1. Differential cross section for elastic scattering by $\mathrm{C}_{2} \mathrm{~F}_{6}$ at (a) 10 , (b) 15, (c) 20, and (d) $30 \mathrm{eV}$. Solid lines are calculated results; circles are experimental values from Ref. 14.

nature of the calculation at hand and taking into account load balance, communication, I/O, and other overhead, we typically see throughput speeds in the range from 5 to 15 GFLOPS. To put these numbers in perspective, our original sequential program, which is only partially vectorizable, averages about 30 MFLOPS on a Y-MP processor.

\section{APPLICATIONS}

In this section we present a selection of results from recent and ongoing calculations of electron scattering by fluorinated hydrocarbons. The systems are chosen to illustrate several points. Elastic scattering by hexafluoroethane, $\mathrm{C}_{2} \mathrm{~F}_{6}$, is an instance where we can compare our results to experimental measurements. ${ }^{14}$ For the difluoromethylene radical, $\mathrm{CF}_{2}$, on the other hand, calculations are no more difficult, and illuminate interesting features of the low-energy scattering, but there are no experimental results. For trifluoromethane or fluoroform, $\mathrm{CHF}_{3}$, we describe how calculations of inelastic scattering cross sections can be used to form estimates of the dissociation cross section.

Figure 1 shows the differential elastic cross section $d \sigma / d \Omega(\theta, E)$ for $\mathrm{C}_{2} \mathrm{~F}_{6}$ at several energies $E$. The data shown by curves in Fig. 1 are taken from recent static-exchange calculations in our group; the circles represent recent measurements. ${ }^{14}$ Overall, there is very satisfactory agreement. Discrepancies in near-forward (small angle) scattering at low energies are due, we believe, to well-understood deficiencies in the static-exchange approximation that can be remedied in a more sophisticated approximation, namely via inclusion of polarization effects (work in progress). A less obvious but equally important issue is the behavior in the backward-scattering (large angle) directions. There is of course no difficulty in calculating cross sections at large 


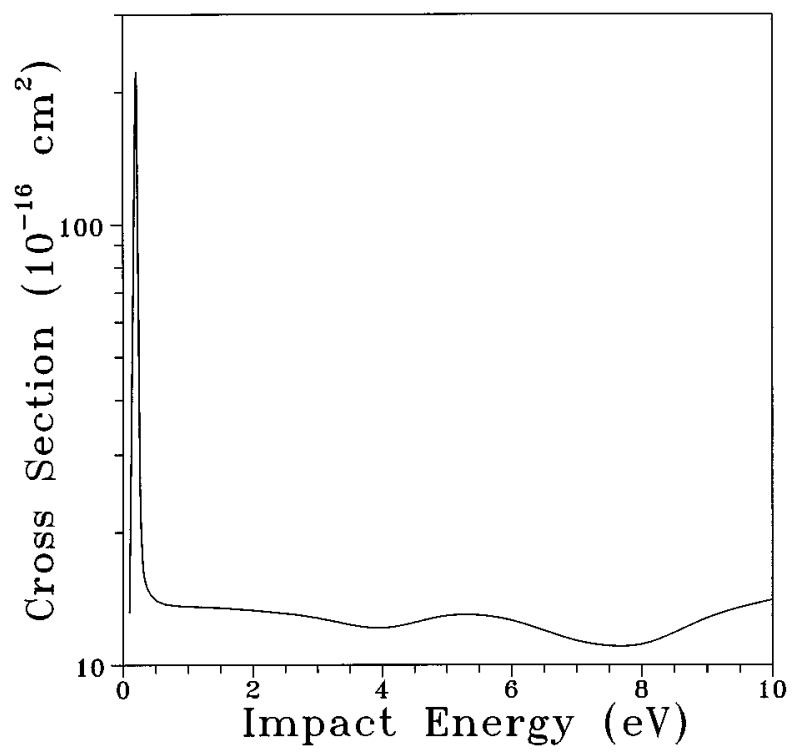

FIG. 2. Calculated integral elastic electron scattering cross section for the $\mathrm{CF}_{2}$ radical.

angles, but measurements must be curtailed at the angle where the detector encounters the electron source. An extrapolation is then necessary to obtain the integral cross section

$$
\sigma_{I}(E)=2 \pi \int d \theta \frac{d \sigma}{d \Omega}(\theta, E),
$$

and the momentum-transfer cross section

$$
\sigma(E)=2 \pi \int d \theta(1-\cos \theta) \frac{d \sigma}{d \Omega}(\theta, E) .
$$

Because of its backward weighting, the latter is especially sensitive to uncertainties in the extrapolation. Our calculations indicate that the reported momentum transfer cross section ${ }^{14}$ is affected significantly by extrapolation error.

As is generally the case for transient species, experimental results are unavailable for the difluoromethylene radical. We are in the process of calculating electron scattering cross sections for $\mathrm{CF}_{2}$; a preliminary result, the integral elastic cross section at low energies, is shown in Fig. 2. In this calculation, we have included polarization effects, which are large at energies below $5 \mathrm{eV}$. Clearly evident as a sharp peak in the cross section is a shape resonance, or temporary negative ion. The resonance is formed by trapping of the scattering electron in the $2 b_{1}$ virtual orbital, which is essentially the out-of-plane C $2 p$ orbital. It should be noted that the calculation is carried out in the fixed-nuclei approximation and at the experimental equilibrium geometry of the neutral radical. The resonance position, height, and width will depend on the nuclear geometry, with the resonance moving to lower energy and becoming stronger and narrower as the $\mathrm{C}-\mathrm{F}$ bond elongates. A complete treatment of this resonance will require that effects of nuclear motion be taken carefully into account, because its lifetime (inversely proportional to its width) is large compared to a vibrational period. Indeed, as

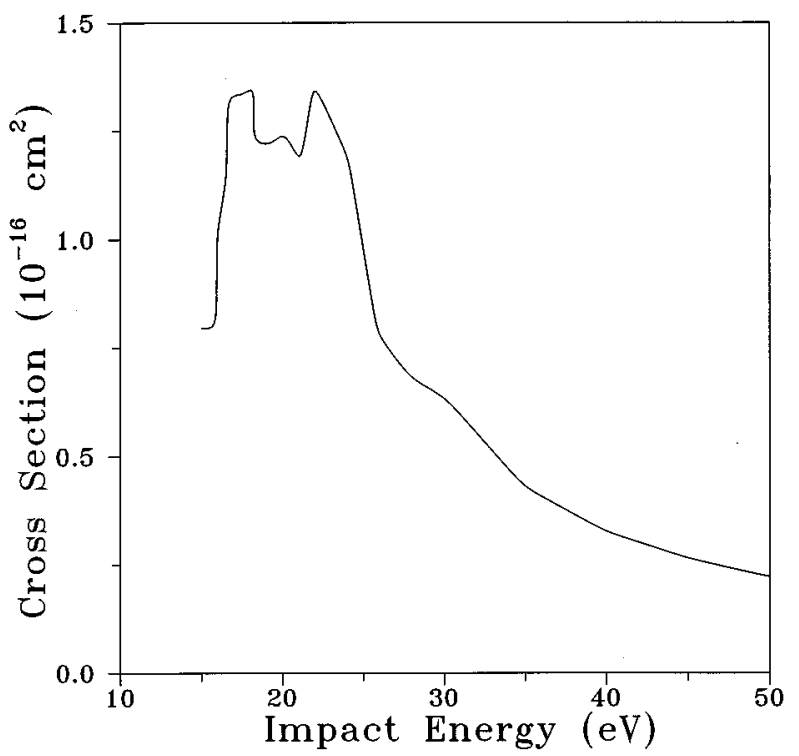

FIG. 3. Estimated cross section for the dissociation process $\mathrm{CHF}_{3} \rightarrow \mathrm{CF}_{3}+\mathrm{H}$ in trifluoromethane, obtained as the sum of four electronic excitation cross sections.

the resonance energy goes to zero, the lifetime becomes infinite, and the resonance evolves into a bound electronic state- the ground state of the $\mathrm{CF}_{2}^{-}$ion. Negative ions formed in this way will be prone to autodetachment but may be stabilized if sufficient vibrational energy is removed in a subsequent collision. This resonant mechanism may be an important contributor to attachment of low-energy electrons by $\mathrm{CF}_{2}$. Calculations such as that illustrated by Fig. 2, while not directly comparable to experiment in the resonance region, provide qualitative information about the attachment process and are the first steps in a quantitative treatment.

Inelastic cross sections, especially those associated with dissociation, are among the most important to plasma modeling. Where it is possible to identify certain electronicexcitation channels as dissociative, we can obtain estimates of the dissociation cross section by summing their cross sections. In the case of $\mathrm{CHF}_{3}$, many of the lowest-lying electronically excited states have potential surfaces on which the $\mathrm{C}-\mathrm{H}$ bond can break, leading to dissociation into $\mathrm{CF}_{3}+\mathrm{H}$. In Fig. 3, we show a preliminary estimate of the cross section for this process, obtained as the sum over four inelastic channels with calculated thresholds between 12.2 and $14.2 \mathrm{eV}$. Certainly more than four channels contribute to dissociation; on the other hand, we have included those channels we believe to be most important, and past experience in other molecules has shown that calculations of this type tend to overestimate the individual channel cross sections; ${ }^{3}$ nor do we expect that fine details of the cross sections, such as threshold behavior, will be correctly reproduced in calculations of this type. We nonetheless expect that the dissociation cross section obtained is semiquantitative: correct in its qualitative features, in particular a broad maximum not far above threshold, and within a factor of 2 or so of the true cross section. It should be noted, however, that the cross section of Fig. 3 
does not yet include corrections for long-range scattering associated with dipole-allowed excitations; these corrections will be fairly minor in the energy range shown, but increasingly important at higher energies. Our calculated results of Fig. 3 differ both qualitatively and quantitatively from the measured $\mathrm{CF}_{3}$ production cross section reported by Sugai et al., ${ }^{15}$ which rises slowly from threshold, with no maximum in the near-threshold region. This difference has significant implications for the neutral fragment production rate because the electron energy distribution function is already small at threshold and is declining exponentially.

\section{CONCLUDING REMARKS}

Continuing rapid advances in the size and power of MPP systems will make it possible to increase both the scale and the sophistication of computational studies of electronmolecule collisions, and to provide reliable data on an ever broader range of molecules and processes. For example, in addition to the studies of fluorocarbons highlighted above, we have also completed extensive studies of electroncollision cross sections for species relevant to $\mathrm{BCl}_{3}$ and $\mathrm{SiCl}_{4}$ etching plasmas. These species included $\mathrm{BCl}_{3}$ and its dissociation fragments $\left(\mathrm{BCl}_{2}\right.$ and $\left.\mathrm{BCl}\right)$ as well as $\mathrm{SiCl}_{4}$ and its fragments $\left(\mathrm{SiCl}_{3}, \mathrm{SiCl}_{2}\right.$, and $\left.\mathrm{SiCl}\right)$. The promise of future MPPs with anticipated performance of many hundreds of GFLOPs lies not only in providing data for the many microscopic processes required in plasma simulations, but in their potential for making possible comprehensive threedimensional simulations of plasma reactors with industrially viable turnaround times. ${ }^{2}$ Such simulations can have significant impact on the time and cost for optimizing reactor design and for characterizing process equipment.

\section{ACKNOWLEDGMENTS}

This work was supported by Sematech, Inc., and by the National Science Foundation's Grand Challenge project on Parallel I/O Methodologies for I/O Intensive Grand Challenge Applications. Use of the computational facilities of the JPL/Caltech Supercomputing Project and of the Center for Advanced Computing Research is gratefully acknowledged.

${ }^{1}$ National Research Council, Plasma Processing of Materials: Scientific Opportunities and Technological Challenges (National Academy Press, Washington, 1991).

${ }^{2}$ S. Shankar, M. Rieffel, S. Taylor, D. Weaver, and A. Wulf, Proceedings of the 12th International Symposium on Plasma Chemistry, Minneapolis, 12-15 August 1995, edited by A. Wendt and I. V. Heberlein, Vol. A, pp. $31-40$.

${ }^{3}$ C. Winstead and V. McKoy Adv. Chem. Phys. 96, 103 (1996).

${ }^{4}$ T. N. Rescigno, B. H. Lengsfield III, and C. W. McCurdy, in Modern Electronic Structure Theory, edited by D. R. Yarkony (World Scientific, Singapore, 1995), Part I, p. 501.

${ }^{5}$ Atomic and Molecular Processes: An R-Matrix Approach, edited by P. G. Burke and K. A. Berrington (Institute of Physics, Bristol, 1993).

${ }^{6}$ C. Winstead and V. McKoy, Adv. At., Mol., Opt. Phys. 36, 183 (1996).

${ }^{7}$ C. Winstead and V. McKoy, in Modern Electronic Structure Theory, edited by D. R. Yarkony (World Scientific, Singapore, 1995), Part II, p. 1375.

${ }^{8}$ C. Winstead, P. G. Hipes, M. A. P. Lima, and V. McKoy J. Chem. Phys. 94, 5455 (1991).

${ }^{9}$ J. Schwinger, Phys. Rev. 72, 742 (1947).

${ }^{10}$ K. Takatsuka and V. McKoy, Phys. Rev. A 30, 1734 (1984); C. Winstead and V. McKoy, ibid. 47, 1514 (1993).

${ }^{11}$ M. A. P. Lima, L. M. Brescansin, A. J. R. da Silva, C. Winstead, and V. McKoy, Phys. Rev. A 41, 327 (1990).

${ }^{12}$ See, e.g., N. S. Ostlund, Chem. Phys. Lett. 34, 419 (1975); D. K. Watson and V. McKoy, Phys. Rev. A 20, 1474 (1979).

${ }^{13}$ C. Winstead, H. P. Pritchard, and V. McKoy, IEEE Comp. Sci. Eng. 2, 34 (1995).

${ }^{14}$ T. Takagi, L. Boesten, H. Tanaka, and M. A. Dillon, J. Phys. B 27, 5389 (1994).

${ }^{15}$ H. Sugai, H. Toyoda, T. Nakano, and M. Goto, Contrib. Plasma Phys. 35, 415 (1995). 